\title{
Ruminação e habilidades de mindfulness em praticantes e não praticantes de meditação
}

\author{
Francisco Crauss \\ Universidade do Vale do Rio dos Sinos, RS, Brasil \\ Carolina Baptista Menezes \\ Universidade do Rio Grande do Sul, RS, Brasil \\ Lucas Nascimento \\ Universidade Federal de Santa Catarina, SC, Brasil \\ Ilana Andretta \\ Universidade do Vale do Rio dos Sinos, RS, Brasil
}

\begin{abstract}
Resumo
Comparou-se os níveis de mindfulness e ruminação entre meditadores e não meditadores entre 16 e 65 anos e investigou-se a correlação dessas variáveis em cada grupo, e com a quantidade de prática. Utilizou-se os questionários sociodemográfico, Ruminação e Reflexão (QRR) e Cinco Facetas de Mindfulness (FFMQ). Os meditadores apresentaram escore significativamente menor de ruminação e maior de mindfulness nas facetas observar, descrever, agir com consciência e não reagir. Agir com consciência, não julgar e não reagir correlacionaram negativamente com ruminação em ambos os grupos, sendo a associação maior entre meditadores. Tempo em meses e frequência semanal correlacionaram positivamente com agir com consciência, não julgar e não reagir e negativamente com ruminação. Os achados corroboram que aumento de mindfulness e redução da ruminação constituem benefícios da prática meditativa, caracterizando potenciais mecanismos psicológicos por trás da relação entre meditação e regulação emocional.
\end{abstract}

Palavras-chave: Meditação; Habilidades de Mindfulness; Ruminação.

\section{Rumination and mindfulness skills in meditation practitioners and non practitioners}

\begin{abstract}
We compared mindfulness and rumination between meditators and non-meditators aging 16-65 years old, and investigated correlations between these variables for each group and their relation to amount of meditative practice. Rumination and Reflection Questionnaire and Five Facets of Mindfulness Questionnaire were used. Meditators had significantly lower scores of rumination and higher scores on the mindfulness facets observe, describe, act with awareness and not react. Act with awareness, not judge and not react negatively correlated with rumination in both groups, but association was stronger for meditators. Time in months and weekly frequency correlated positively with facets of mindfulness and negatively with rumination. Results support that meditation may foster mindfulness and reduced rumination, and corroborate the understanding that these changes are potential psychological mechanisms underlying the relationship between meditation and emotional regulation.
\end{abstract}

Keywords: Meditation; Mindfulness Skills; Rumination.

\section{Rumia y habilidades de mindfulness en practicantes de la meditación y no practicantes}

\section{Resumen}

Comparamos mindfulness y la rumia entre meditadores y no meditadores, y investigamos las correlaciones entre estas variables para cada grupo y su relación con la cantidad de práctica meditativa. Os cuestionario Rumination y Reflection, y Five Facets of Mindfulness fueron utilizados. Los meditadores presentaron puntuaciones más bajas de rumia y más altas en las facetas de mindfulness observar, describir, actuar con conciencia y no reaccionar. Actuar con conciencia, no juzgar y no reaccionar correlacionaron con la rumia en ambos grupos, pero la asociación fue más fuerte para meditadores. El tiempo en meses y la frecuencia semanal correlacionaron positivamente con las facetas de mindfulness y negativamente con la rumia. Los resultados apoyan que la meditación puede fomentar mindfulness y reducir la rumia, y corroboran la comprensión de que estos cambios son potenciales mecanismos psicológicos subyacentes a la relación entre la meditación y la regulación emocional.

Palabras clave: Meditación; Habilidades de Mindfulness; Ruminación. 


\section{Introdução}

\section{Meditação e ruminação}

A ruminação é uma resposta ao estresse psicológico que envolve um foco repetitivo e persistente nos sintomas do estresse e sentimentos negativos, bem como nas possíveis causas e consequências negativas desses sintomas. Desse modo, a ruminação acaba tornando-se um fator de risco para o agravamento do estresse ou para o desenvolvimento de problemas psicológicos, muitas vezes ocasionando um ciclo ruminativo (Treynor, Gonzalez, \& Nolen-Hoeksema, 2003). Neste sentido, é importante investigar estratégias que auxiliem na redução da ruminação.

Estudos têm demonstrado que a prática de meditação pode ser uma alternativa para a redução dos níveis de estresse e de ruminação, sendo que tanto a indução de um estado meditativo (uma sessão de meditação), como treinos mais longos de meditação (Wolkin, 2015) parecem influenciar estes desfechos. Por exemplo, em uma amostra de adultos saudáveis, uma breve indução da meditação mindfulness (focada na respiração) ajudou a reduzir a frequência de pensamentos negativos após a exposição a estímulos emocionais negativos, comparado ao grupo controle (Kiken \& Shook, 2014). Outro estudo com desenho experimental semelhante mostrou que o grupo que também passou por uma breve prática da meditação da respiração apresentou uma redução da recordação de palavras de valência negativa em um teste de memória, comparado ao grupo controle (Alberts \& Thewissen, 2011). Em consonância com estes achados, uma prática breve da mesma meditação realizada após uma indução experimental de humor negativo ajudou participantes universitários saudáveis a reduzirem os níveis de ruminação estado (Hilt \& Pollak, 2012).

Intervenções mais longas com amostras clínicas também parecem produzir efeitos significativos na redução da ruminação. Por exemplo, foi demonstrado que mulheres com câncer que participaram do programa Mindfulness-Based Stress Reduction (MBSR; 8 semanas) reduziram os níveis de ruminação em comparação a um grupo de lista de espera (Campbell, Labelle, Bacon, Faris, \& Carlson, 2012). No estudo de Ramel, Goldin, Carmona e McQuaid (2004), o MBSR gerou uma diminuição no pensamento ruminativo em pessoas com história de transtornos afetivos, relativo ao grupo controle, sendo que uma maior quantidade de prática se relacionou a menores níveis de ruminação.

Os mesmos efeitos são observados em amostras não clínicas. Em uma pesquisa que avaliou o efeito do MBSR em universitários com queixa de estresse, os resultados apontaram uma redução significativa do estresse no grupo experimental, a qual foi mediada pela redução dos níveis de ruminação (Jain et al., 2007). O mesmo programa também produziu uma redução dos escores de ruminação apenas no grupo experimental, mas neste caso em uma amostra de jovens adultos sem queixa específica de estresse (Chambers, Yee Lo, \& Allen, 2008). Neste estudo, o programa também produziu um aumento dos escores de mindfulness, sendo que houve uma correlação entre menores índices de ruminação e maiores índices de mindfulness. Portanto, a ruminação parece envolver um estilo de pensamento e de atividade mental inverso ao padrão da prática meditativa e ao cultivo de mindfulness.

\section{Meditação e habilidades de mindfulness}

Apesar de existirem diferentes estilos de práticas de meditação, tal como atenção focada (também chamada de concentrativa) e monitoramento aberto (também chamada de mindfulness; para uma maior discussão sobre os diferentes tipos, ver Dahl, Lutz, \& Davidson, 2015; Lutz, Dunne, \& Davidson, 2007; Menezes \& Dell'Aglio, 2009), entende-se que um objetivo comum a todas é o desenvolvimento de uma consciência menos condicionada, menos distraída e menos reativa (Walsh \& Shapiro, 2006). Portanto, tanto do ponto de vista das filosofias tradicionais, como da ciência psicológica, as práticas meditativas buscam cultivar e desenvolver mindfulness, isto é, uma habilidade mental que auxilia o praticante a controlar a automaticidade com que tende a realizar julgamentos e a reagir afetivamente às próprias associações, sensações e sentimentos (Brown \& Ryan, 2003).

Embora o termo mindfulness seja comumente utilizado para designar um estilo de prática meditativa (Bishop et al., 2004), é importante salientar que o mesmo também é utilizado para referir-se à própria qualidade mental (ou característica psicológica) que as práticas meditativas buscam desenvolver e aprimorar (Brown \& Ryan, 2003; Sauer et al., 2013). No português, não existe uma tradução literal para o termo, sendo a expressão "atenção plena" a mais utilizada para representar este conceito (Menezes, Dell'Aglio, \& Bizarro, 2012; Vandenberghe \& Assunção, 2009). Assim, na condição de prática meditativa, mindfulness é muitas vezes traduzido como uma "prática de atenção plena", enquanto na condição de qualidade mental, mindfulness designa um traço e/ou estado psicológico de "atenção plena no momento presente".

No âmbito da investigação científica de mindfulness enquanto uma qualidade mental, evidências sugerem que o construto é multifatorial, ou seja, representado por um conjunto de habilidades interrelacionadas. Por 
isso, os termos "traço mindfulness (ou "mindfulness disposicional") e "habilidades de mindfulness" têm sido utilizados intercambiavelmente (Baer, Smith, Hopkins, Krietemeyer, \& Toney, 2006; mas ver Siegling \& Petrides, 2014). Em um estudo que avaliou a estrutura fatorial de cinco questionários que mensuram mindfulness (Baer et al., 2006), cinco facetas se destacaram e se distinguiram, melhor descrevendo e representando o construto: observar, descrever, agir com consciência, não julgar e não reagir. Deste estudo surgiu o Five Facet Mindfulness Questionnaire (FFMQ) (Baer et al., 2008), um dos instrumentos de mensuração psicométrica de mindfulness mais utilizado mundialmente (Sauer et al., 2013), já validado para o Brasil (Barros, Kozasa, Souza, \& Ronzani, 2014).

No estudo original de construção e validação do FFMQ (Baer et al., 2008), exceto a faceta agir com consciência, as outras quatro dimensões relacionaramse com a experiência de meditação e mediaram a relação entre experiência de meditação e bem-estar psicológico. Recentemente, outro estudo comparou as facetas do FFMQ entre uma ampla amostra de meditadores $(n=384)$ e não meditadores $(n=286)$ (Soler et al., 2014). O grupo da meditação apresentou escores significativamente mais altos para as cinco facetas de mindfulness, embora os tamanhos de efeito tenham sido maiores para as facetas observar e não reagir. Além disso, este estudo também encontrou que os níveis mais altos de mindfulness relacionaram-se com o maior tempo em meses e a maior frequência semanal de prática, sem sofrer influência da duração da prática (minutos por sessão) ou do tipo de meditação. Em outra pesquisa que também comparou o FFMQ entre amostras igualmente grandes de meditadores $(n=288)$ e não meditadores $(n=451)$, os escores de todas as facetas de mindfulness foram significativamente mais altos no grupo de praticantes (de Bruin, Topper, Muskens, Bögels, \& Kamphuis, 2012). O mesmo padrão de resultado foi encontrado no estudo de validação do FFMQ para o Brasil (Barros et al., 2014).

\section{Ruminação e habilidades de mindfulness}

De forma geral, mesmo em contextos não meditativos, a ruminação e as habilidades de mindfulness parecem representar estilos distintos e inversos de processamento. Por exemplo, um estudo investigou o efeito das habilidades de mindfulness (indexadas pelo FFMQ) e da ruminação no desempenho de universitárias mulheres saudáveis em um paradigma que avaliava a tolerância ao estresse (Feldman, Dunn, Stemke, Bell, \& Greeson, 2014). Enquanto a ruminação correlacionou-se com maiores níveis de afeto negativo relatado após a tarefa, as facetas não julgar e não reagir correlacionaram-se com menor nível de afeto negativo relatado após a tarefa. Estas facetas do FFMQ também predisseram a persistência em uma tarefa com anagramas de alta dificuldade em outro estudo que avaliou uma amostra universitária saudável majoritariamente feminina (Evans, Baer, \& Segerstrom, 2009). Além disso, os maiores níveis na faceta não julgar foram um preditor significativo de menores níveis de ruminação em uma amostra de universitários saudáveis, mesmo controlando para o efeito do afeto negativo (Kiken \& Shook, 2014). Em uma amostra masculina, a ruminação relacionou-se de forma inversa e significativa especificamente com a faceta não reagir do FFMQ após os participantes terem passado por uma indução de estresse (Paul, Stanton, Greeson, Smoski, \& Wang, 2013).

Considerando o contexto meditativo, outras pesquisas corroboram o estudo já citado de Chambers e colaboradores (2008), no qual foi encontrada uma relação inversa entre ruminação e mindfulness. No estudo de de Bruin e colaboradores (2012), foi observada uma correlação negativa significativa entre quatro facetas do FFMQ (descrever, agir com consciência, não julgar e não reagir) e os escores de ruminação em meditadores experientes. Similarmente, o escore geral de mindfulness da Escala de Atenção e Consciência Plena (MAAS) também correlacionouse negativamente com os escores de ruminação em praticantes (Brown \& Ryan, 2003).

Apesar das evidências apoiarem os efeitos da meditação na redução da ruminação e no aumento de habilidades de mindfulness, bem como a relação inversa entre níveis de mindfulness e níveis de ruminação, nenhum estudo avaliou estas variáveis conjuntamente comparando meditadores experientes e não meditadores. Assim, este estudo buscou avaliar se estes efeitos e correlações ocorrem quando meditadores experientes e não meditadores são comparados. Além disso, no grupo dos meditadores buscou-se avaliar o efeito da quantidade de prática nestes desfechos. Este estudo esperava encontrar níveis significativamente menores de ruminação e maiores de mindfulness nos praticantes de meditação em comparação aos não praticantes. Também esperava observar uma correlação significativa destes desfechos com a quantidade de prática dos meditadores; assim como uma correlação negativa significativa entre os escores de mindfulness e de ruminação para ambos os grupos, mas com uma força de associação maior para o grupo da meditação. 


\section{Método}

\section{Participantes}

Participaram deste estudo 201 pessoas entre $16 \mathrm{e}$ 65 anos, com idade média de 36.42 anos ( $\mathrm{DP}=11.48$ ), de ambos os sexos, divididas em dois grupos: 1) 78 pessoas que praticavam meditação sentada e silenciosa de forma individual ou em grupo, há pelo menos um mês, sendo que poderiam ser praticantes tanto de meditação da atenção focada, como de monitoramento aberto; e 2) 123 pessoas que não praticavam meditação. A amostra foi selecionada por conveniência através de divulgação nas redes sociais e grupos de interesse no tema. No texto de divulgação para selecionar as pessoas do grupo de meditação, era informado que estavam sendo selecionados apenas os praticantes que realizavam meditação sentada e silenciosa dos tipos atenção focada e monitoramento aberto, sendo que uma descrição de cada técnica era fornecida.

\section{Instrumentos}

Foi utilizado um Questionário Sociodemográfico especificamente para este estudo, que investigou os dados: idade, grau de escolaridade, estado civil, renda mensal, uso de medicação psiquiátrica, acompanhamento psicoterapêutico, e dados da experiência de prática meditativa. Devido um erro na formatação do instrumento, a variável sexo não foi incluída.

Para avaliar os níveis de pensamentos ruminativos, foi aplicado o Questionário de Ruminação e Reflexão (QRR), que se trata de um questionário de autorrelato composto por duas escalas de 12 itens cada (12 referentes à ruminação e 12 à reflexão), respondido através de uma escala Likert de 5 pontos. Para este estudo, foi utilizada apenas a escala de ruminação, a qual avalia o quanto indivíduos engajam em pensamentos ruminativos (p.e., "Eu sempre pareço estar remoendo em minha mente coisas recentes que disse ou fiz"). A subescala de ruminação apresenta adequada consistência interna, com índice alpha de Cronbach de 0.87 no estudo brasileiro (Zanon \& Teixeira, 2006).

O Questionário das Cinco Facetas de Mindfulness (FFMQ-BR) (Five Facet Mindfulness QuestionnaireFFMQ (Barros et al., 2014) é composto por 39 itens de autorrelato respondidos numa escala de tipo Likert de 1 a 5, os quais avaliam cinco habilidades de mindfulness, bem como o escore geral, $\mathrm{O}$ instrumento foi adaptado e validado para o Brasil por Barros et al (2014). As subescalas obtiveram os seguintes índices de consistência interna: não julgar $(\alpha=0.78)$, agir com consciência $(\alpha=0.79)$, observar $(\alpha=0.76)$, descrever $(\alpha=0.76)$, e não reagir $(\alpha=0.68)$.

\section{Procedimentos}

O projeto de pesquisa foi encaminhado ao Comitê de Ética da UNISINOS para apreciação e avaliação sob o parecer número13/115, de acordo as Diretrizes e Normas Regulamentadoras para pesquisa com seres humanos, previstas na Resolução no 196/1996, do Conselho Nacional de Saúde e na Resolução no 016/2000 do Conselho Federal de Psicologia. Este estudo tomou todos os cuidados éticos necessários, de modo a garantir o anonimato no tratamento dos dados e divulgação dos achados dessa pesquisa, bem como o princípio de não causar dano aos participantes. Os participantes foram instruídos que caso sentissem algum desconforto ao responder algum questionário, poderiam interromper o preenchimento e contatar o pesquisador através do telefone ou e-mail para possíveis orientações e, caso necessário, encaminhamentos seriam feitos. Eles tinham a opção de desistir da participação da pesquisa caso julgassem necessário, sem que isso acarretasse qualquer prejuízo. Aqueles que desejassem uma devolução dos dados poderiam solicitar ao avaliador por telefone ou e-mail.

Foi elaborado um formulário de preenchimento na ferramenta Google Docs, com todos os instrumentos descritos acima para autopreenchimento. Antes de iniciar o preenchimento dos instrumentos da pesquisa, todos tiveram que assinar o Termo de Consentimento Livre e Esclarecido via web e só puderam dar continuidade após a confirmação da leitura e da aceitação dos termos do TCLE. Foi necessário que o participante preenchesse todos os dados pessoais para avançar no preenchimento. O formulário foi divulgado via redes sociais para a coleta de dados da amostra de ambos os grupos. Os participantes foram instruídos a preenchêlos em um momento de calma e silêncio, para haver o mínimo de interferência ambiental na sua atenção. Cada página apresentada continha as questões referentes a um instrumento e, após o preenchimento de todas as questões, o participante tinha a opção de passar para o próximo instrumento.

\section{Análise dos dados}

Inicialmente foram utilizadas médias (desvio padrão) ou porcentagens para descrever as variáveis sociodemográficas (idade, escolaridade, estado civil, renda, uso de medicação, tratamento psicoterápico) e de prática de meditação (tempo em meses, regularidade semanal, duração em minutos). As variáveis sociodemográficas foram então comparadas entre os grupos de praticantes de meditação e não praticantes através das análises de qui-quadrado $\left(\chi^{2}\right)$ e teste $t$ de Student. Posteriormente, as Análises de Variância Multivariada 
(MANOVA) e Univariada (ANOVA) foram utilizadas para comparar os domínios do FFMQ (observar, descrever, agir com consciência, não julgar, não reagir) entre os grupos, e a ANOVA foi utilizada para comparar a ruminação, controlando para idade. Por fim, utilizou-se a análise de Correlação de Pearson Parcial para avaliar as correlações entre as variáveis dentro de cada grupo, controlando para idade, sendo que apenas no grupo da meditação também foram controladas as variáveis de tempo e frequência semanal de prática. A Correlação de Pearson Bivariada foi utilizada para avaliar as correlações entre os desfechos e as variáveis de quantidade de prática de meditação no grupo de meditadores. Para todas as análises utilizou-se o SPSS 20.0, adotando um nível de significância bicaudal igual ou menor que .05 .

\section{Resultados}

A Tabela 1 apresenta a caracterização sociodemográfica da amostra composta por 201 respondentes. Conforme pode ser observado, não houve diferença estatisticamente significativa entre os grupos para nenhuma das variáveis medidas. Nesta tabela também constam os dados descritivos da prática de meditação para o grupo de meditadores.

Os dados descritivos, bem como as estatísticas dos testes realizados para cada desfecho encontramse na Tabela 2. Com relação à ruminação, o grupo de meditadores relatou um escore significativamente menor $\left(p=.001, \eta_{p}{ }^{2}=.05\right)$ em comparação aos não meditadores. Referente ao FFMQ, a comparação multivariada foi significativa [Box's $M=15.94, p=.41$; Lambda de Wilk=.900, $F(5,195)=4.34, p=.001$, $\eta_{p}{ }^{2}=.10$; Levene's $\left.F p>.10\right]$, sendo que de acordo com as análises univariadas, o grupo de meditadores apresentou um escore significativamente maior para os domínios observar $\left(p=.004, \eta_{p}^{2}=.04\right)$, descrever $\left(p=.006, \eta_{p}^{2}=.04\right)$, agir com consciência $(p=.003$, $\left.\eta_{p}{ }^{2}=.04\right)$ e não reagir $\left(p=.000, \eta_{p}^{2}=.06\right)$. Não houve diferença significativa para não julgar $(p=.48$, $\eta_{p}^{2}=.002$ ).

Nas análises de Correlação de Pearson, o mesmo padrão de correlações negativas entre ruminação e os domínios de agir com consciência, não julgar e não reagir foram observados para ambos os grupos, no entanto a força de associação foi maior para o grupo de meditadores. Todos os valores das correlações e da significância estatística para cada grupo estão descritos na Tabela 3.

Por fim, foi possível observar que dentro do grupo de meditadores, tanto o tempo de prática em meses, como a frequência semanal, se correlacionaram positivamente com os domínios agir com consciência, não julgar e não reagir e negativamente com a ruminação. Não houve correlação significativa entre a duração da prática em minutos e nenhum dos domínios. Todos os valores podem ser observados na Tabela 4.

TABELA 1

Comparação das Variáveis do Questionário Sociodemográfico entre os Grupos.

\begin{tabular}{|c|c|c|c|c|c|}
\hline \multirow{3}{*}{ Variáveis } & \multicolumn{5}{|c|}{ Grupo } \\
\hline & \multicolumn{2}{|c|}{$\begin{array}{c}\text { Meditadores } \\
\quad(n=78)\end{array}$} & \multicolumn{2}{|c|}{$\begin{array}{c}\text { Não-Meditadores } \\
\quad(n=123)\end{array}$} & \multirow[t]{2}{*}{$p$} \\
\hline & $N$ & Мои \% & $N$ & Мои \% & \\
\hline \multicolumn{5}{|l|}{ Idade $^{\mathrm{a}, \mathrm{b}}$} & .66 \\
\hline Média (DP) & 78 & $36.87(12.53)$ & 123 & $36.14(10.8)$ & \\
\hline Amplitude & & $(16-65)$ & & $(22-65)$ & \\
\hline \multicolumn{5}{|l|}{ Escolaridade*a,b } & .33 \\
\hline Fundam. Incompleto & 0 & 0 & 2 & 1.6 & \\
\hline Fundam. Completo & 0 & 0 & 2 & 1.6 & \\
\hline Médio Incompleto & 3 & 3.8 & 1 & 0.8 & \\
\hline Médio Completo & 7 & 9 & 8 & 6.5 & \\
\hline Superior Incompleto & 21 & 26.9 & 28 & 22.8 & \\
\hline Superior Completo & 47 & 60.3 & 82 & 66.7 & \\
\hline \multicolumn{5}{|l|}{ Estado civil*a,b } & .10 \\
\hline Solteiro & 29 & 37.2 & 52 & 42.3 & \\
\hline Casado/União Estável & 32 & 41 & 59 & 48 & \\
\hline Separado/Divorciado & 16 & 20.5 & 12 & 9.8 & \\
\hline Viúvo(a) & 1 & 1.3 & 0 & 0 & \\
\hline \multicolumn{5}{|l|}{ Renda Mensala ${ }^{a, b}$} & .56 \\
\hline < 1 salário mínimo & 7 & 9 & 6 & 4.9 & \\
\hline 1-5 salários mínimos & 33 & 42.3 & 60 & 48.8 & \\
\hline 5-10 salários mínimos & 23 & 29.5 & 37 & 30.1 & \\
\hline 10-15 salários mínimos & 8 & 10.3 & 14 & 11.4 & \\
\hline > 15 salários mínimos & 7 & 9 & 6 & 4.9 & \\
\hline \multicolumn{5}{|l|}{ Medicação psiquiátricaa,b } & .14 \\
\hline Sim & 17 & 21.8 & 17 & 13.8 & \\
\hline Não & 61 & 78.2 & 106 & 86.2 & \\
\hline \multicolumn{5}{|l|}{ Faz Psicoterapia ${ }^{\mathrm{a}, \mathrm{b}}$} & .49 \\
\hline Sim & 20 & 25.6 & 37 & 30.1 & \\
\hline Não & 58 & 74.4 & 86 & 69.9 & \\
\hline \multicolumn{6}{|l|}{ Tempo prática (meses) } \\
\hline Média (DP) & \multirow{2}{*}{\multicolumn{4}{|c|}{ 45.83(51.35) }} & \\
\hline Amplitude & & & & & \\
\hline \multicolumn{6}{|l|}{ Frequência (semanal) } \\
\hline Média (DP) & \multicolumn{3}{|c|}{$2.9(2.3)$} & & \\
\hline Amplitude & \multicolumn{3}{|c|}{$(0.25-7)$} & & \\
\hline \multicolumn{6}{|l|}{ Duração da prática (min) } \\
\hline Média (DP) & \multirow{2}{*}{\multicolumn{3}{|c|}{$\begin{array}{c}35.96(31.7) \\
(3-180)\end{array}$}} & & \\
\hline Amplitude & & & & & \\
\hline
\end{tabular}

* Dados faltantes.

a Teste $t$ de Student.

b Teste qui-quadrado. 
TABELA 2

Análise comparativa univariada dos testes QRR e FFMQ entre os Grupos.

\begin{tabular}{|c|c|c|c|c|c|}
\hline \multirow{2}{*}{ Variáveis } & \multirow{2}{*}{ Pratica meditação? } & \multirow{2}{*}{$M(D P)$} & \multicolumn{3}{|c|}{ ANOVA } \\
\hline & & & $F$ & $p$ & $\eta_{p}{ }^{2}$ \\
\hline \multirow[t]{2}{*}{ Ruminação } & Sim & $2.93(.10)$ & 11.48 & $.001 *$ & .05 \\
\hline & Não & $3.36(.07)$ & & & \\
\hline \multicolumn{6}{|l|}{ FFMQ } \\
\hline \multirow[t]{2}{*}{ Observar } & Sim & $3.74(.09)$ & 8.7 & $.004 *$ & .04 \\
\hline & Não & $3.39(.07)$ & & & \\
\hline \multirow[t]{2}{*}{ Descrever } & Sim & $3.82(.09)$ & 7.63 & $.006 *$ & .03 \\
\hline & Não & $3.50(.07)$ & & & \\
\hline \multirow[t]{2}{*}{ Agir com consciência } & Sim & $3.41(.07)$ & 8.96 & $.003 *$ & .04 \\
\hline & Não & $3.11(.06)$ & & & \\
\hline \multirow[t]{2}{*}{ Não julgar } & Sim & $2.94(.10)$ & .49 & .484 & .002 \\
\hline & Não & $2.85(.08)$ & & & \\
\hline \multirow[t]{2}{*}{ Não reagir } & Sim & $3.19(.07)$ & 13.69 & $.000 *$ & .06 \\
\hline & Não & $2.81(.06)$ & & & \\
\hline
\end{tabular}

* Dados em negrito, indicando $p<0,05$ ou $p<0,001$.

TABELA 3

Correlações entre os testes QRR e FFMQ para cada Grupo.

\begin{tabular}{lcccccccccccc}
\hline & \multicolumn{3}{c}{ Observar } & \multicolumn{2}{c}{ Descrever } & \multicolumn{2}{c}{ Agir com consciência } & \multicolumn{2}{c}{ Não julgar } & \multicolumn{2}{c}{ Não reagir } \\
Pratica meditação? & Sim & Não & Sim & Não & Sim & Não & Sim & Não & Sim & Não \\
\hline Ruminação & -.19 & .06 & -.18 & -.14 & -.53 & -.39 & -.53 & -.46 & -.63 & -.32 \\
$p$ & .09 & .45 & .10 & .11 & $\mathbf{< . 0 0 1 *}$ & $<.001 *$ & $<.001 *$ & $<.001 *$ & $<.001 *$ & $<.001 *$ \\
\hline
\end{tabular}

* Dados em negrito, indicando $p<0,05$ ou $p<0,001$.

TABELA 4

Correlações entre Experiência de Meditação e os Testes QRR e FFMQ.

\begin{tabular}{|c|c|c|c|c|c|c|}
\hline Prática & Observar & Descrever & Agir com consciência & Não julgar & Não reagir & Ruminação \\
\hline Tempo (meses) & .04 & .03 & .31 & .27 & .29 & -.30 \\
\hline$p$ & .71 & .76 & $<.001 *$ & $.02 *$ & $.01 *$ & $<.001 *$ \\
\hline Frequência (semanal) & .19 & .16 & .22 & .22 & .28 & -.27 \\
\hline$p$ & .08 & .14 & $.05 *$ & $.05 *$ & $.01 *$ & $.01 *$ \\
\hline Duração (min) & .08 & -.18 & .04 & .18 & .00 & -.06 \\
\hline$p$ & .44 & .12 & .68 & .11 & .99 & .55 \\
\hline
\end{tabular}

* Dados em negrito, indicando $p<0,05$ ou $p<0,001$.

\section{Discussão}

Este estudo objetivou comparar praticantes e não praticantes de meditação com relação aos seus níveis autorrelatados de habilidades de mindfulness (observar, descrever, agir com consciência, não julgar e não reagir) e ruminação. Além disso, buscou-se investigar a relação entre estas variáveis para cada grupo separadamente, bem como a relação destas variáveis com a quantidade de prática de meditação. Foi observado que em comparação aos não praticantes, os meditadores relataram escores significativamente maiores para quatro facetas do FFMQ, a saber, observar, descrever, agir com consciência e não reagir, e significativamente menores para o estilo de pensamento ruminativo, mesmo controlando para idade. Assim, parece que 
em comparação aos não praticantes, os meditadores apoiam-se com maior frequência e/ou maior facilidade nas habilidades de observação (perceber e identificar os estímulos internos e externos com maior facilidade), descrição (discernir os estímulos com maior clareza), de agir com consciência (prestar atenção nas atividades sendo realizadas), de ser menos reativo (não reagir às experiências internas de forma automática, agindo com postura de observador) e conseguem ter maior controle da tendência de ruminar, isto é, de focalizar a atenção de forma repetitiva em sentimentos e eventos considerados desagradáveis ou ameaçadores.

Embora este seja um estudo transversal, não permitindo inferências sobre relações de causalidade, as diferenças observadas vão ao encontro de outros estudos que utilizaram uma metodologia semelhante (de Bruin et al., 2012; Soler et al., 2014). Entendese que a convergência destes resultados transversais remete a duas hipóteses: 1 . as pessoas que meditam compartilham características comuns independente da prática propriamente dita (podendo até mesmo procurar este tipo de prática em função destas características); ou 2. a prática de meditação de fato contribui para aumentar os níveis de mindfulness e reduzir o estilo cognitivo de ruminação. Apesar de o presente estudo não ter testado estas hipóteses diretamente, sugere-se que a segunda hipótese é parcialmente apoiada pelas correlações encontradas, as quais apontaram uma associação da quantidade de prática (tempo de prática em meses e frequência semanal) com os escores mais alto nas facetas agir com consciência, não julgar e não reagir; bem como com escores menores de ruminação. Portanto, é possível que a diferença encontrada entre os grupos seja devido ao efeito que a prática de meditação de fato pode exercer sobre mindfulness e ruminação. Além disso, estas correlações convergem com outros estudos transversais que também encontraram que o tempo em meses e a frequência semanal foram mais preditivos para os desfechos avaliados, tal como maiores níveis de mindfulness (Soler et al., 2014) e bem-estar (Menezes \& Dell'Aglio, 2010), e menores níveis de ruminação (Ramel et al., 2004).

A hipótese de que os resultados observados podem ser consequência da prática também é corroborada por outros estudos que avaliaram intervenções com meditação longitudinalmente. Existem evidências do aumento nos escores de mindfulness, bem como da redução nos escores de ruminação, em pessoas que participaram de treinos meditativos de oito semanas, em comparação a quem não recebeu intervenção (Brown \& Ryan, 2003; Chambers et al., 2008; Jain et al., 2007).
Discute-se que um dos efeitos da meditação é a modificação de estilos cognitivos, isto é, da forma como a pessoa processa e relaciona-se com seus próprios conteúdos mentais e suas próprias experiências (Brown \& Ryan, 2003; Menezes et al., 2012; Walsh \& Shapiro, 2006). Assim, é possível que a meditação auxilie a modificar estilos cognitivos através de uma relação inversa entre um padrão ruminativo e um padrão mindful (de atenção plena). Esta ideia é corroborada pelas correlações negativas encontradas neste estudo entre ruminação e três facetas de mindfulness, a saber, agir com consciência, não julgar e não reagir, sendo que no grupo de meditadores as associações foram mais fortes especialmente para duas das facetas que os diferiram dos não meditadores (agir com consciência e não reagir). Portanto, desenvolver o controle do "piloto automático" pode facilitar que os indivíduos interrompam a tendência de constantemente contemplar problemas e situações de conflito ou estresse, e atenuem suas reações afetivas a esses fenômenos mentais.

Não obstante, destaca-se que mesmo com um efeito menor, estas correlações também foram significativas no grupo de não meditadores. Dessa forma, esses resultados apoiam estudos que encontraram uma relação inversa entre mindfulness e ruminação tanto em meditadores (Brown \& Ryan, 2003; Chambers et al., 2008; de Bruin et al., 2012; Nyklíček \& Kuijpers, 2008), como não meditadores (de Bruin et al., 2012; Kiken \& Shook, 2014; Paul et al., 2013), sugerindo que há uma interdependência destes padrões mentais para o funcionamento psicológico, e que esta interdependência pode ser especialmente trabalhada no contexto da meditação.

Portanto, tomadas em conjunto, diversas evidências convergentes - tanto no que tange aos resultados do presente estudo, como a consonância desses com outros estudos - apontam que a prática da meditação pode ajudar a aumentar habilidades de mindfulness e a diminuir um estilo de pensamento ruminativo. No entanto, alguns resultados também apresentam algum grau de divergência. Por exemplo, existem evidências de que a prática meditativa relaciona-se com maiores escores nas cinco facetas de mindfulness (Barros et al., 2014; de Bruin et al., 2012; Soler et al., 2014), embora em um dos estudos o efeito observado tenha sido maior para observação e não reagir (Soler et al., 2014). Já no estudo de Baer et al. (2008), apenas agir com consciência não relacionou-se de forma significativa com a experiência em meditação. No presente estudo, os grupos apenas não diferiram na faceta não julgar, e o maior tamanho de efeito foi para não reagir (.06), seguido de observar (.04), agir com consciência (.04) e descrever (.03). 
Ainda, vale destacar que no estudo de validação Brasileira do FFMQ, embora meditadores tenham apresentado maiores níveis de mindfulness para todas as facetas relativo a não meditadores, a amostra de meditadores tinha apenas 36 participantes e os tamanhos de efeito não foram reportados no trabalho (Barros et al., 2014). Além disso, uma limitação comum ao presente estudo e ao estudo de Barros et al. (2014) é que a mensuração e o controle da distribuição dos tipos de meditação não foram feitos (mesmo que o presente estudo tenha avaliado apenas a meditação sentada e silenciosa), inviabilizando discutir se as diferenças observadas podem variar conforme o tipo de prática sendo avaliada. Não obstante, no estudo de Soler et al. (2014), o tipo de meditação foi mensurado e esta variável não moderou as diferenças detectadas entre meditadores e não meditadores, tampouco influenciou os tamanhos de efeito observados. Além disso, uma meta-análise mostrou que a variável "tipo de meditação" também não moderou os efeitos psicológicos positivos da meditação, em particular emocionais e cognitivos (Sedlmeier et al., 2012).

Assim, sugere-se duas hipóteses não necessariamente excludentes para explicar a inconsistência de resultados sobre habilidades de mindfulness: 1) por um lado, uma potencial influência da heterogeneidade das amostras, dos delineamentos e até mesmo dos instrumentos utilizados; 2) por outro, a ideia de que mindfulness de fato compreende um construto multifatorial, de forma que mesmo no contexto da meditação, as diferentes facetas que o compõe podem se manifestar e se combinar de formas distintas e em diferentes graus. Esta hipótese estaria em consonância com a observação de que mesmo com algumas divergências, de modo mais abrangente parece ser consistente a associação da prática de meditação com pelo menos alguma(s) habilidade(s) de mindfulness.

Por fim, além das limitações já ponderadas ao longo da discussão, é importante destacar outros fatores que podem ser potencialmente limitantes deste estudo. Por uma falha técnica na formatação do instrumento, a variável sexo não foi mensurada, não sendo possível testar seu efeito moderador. Ademais, apesar de ter havido uma comparação dos grupos com relação ao uso de medicamentos psiquiátricos e tratamento psicoterápico, a presença de transtornos psiquiátricos não foi avaliada e controlada nas análises. Além disso, considerando que a variável ruminação está diretamente relacionada ao estresse psicológico, outra limitação deste estudo foi a não mensuração da frequência de eventos estressores, especialmente no período das avaliações.

\section{Conclusão}

Os achados indicaram que meditadores apresentaram um maior nível das habilidades de mindfulness do FFMQ (exceto não julgar) e menor ruminação, sendo que os níveis de agir com consciência, não julgar e não reagir foram especialmente maiores para quem relatou maior tempo e frequência de prática. Além disso, nos meditadores houve uma associação negativa mais forte entre as habilidades agir com consciência, não julgar e não reagir e ruminação. Estes achados estão de acordo com a ideia de que a meditação ajuda os praticantes a desenvolverem uma maior consciência sobre seus padrões mentais, mudando a forma como reagem às emoções, sensações e/ou pensamentos que são normalmente evocados por tais padrões. Por fim, a relevância destes resultados está especialmente atrelada ao fato de que tanto o aumento de mindfulness, quanto a redução da ruminação, parecem caracterizar mecanismos psicológicos por trás da relação entre meditação, bem-estar e regulação emocional (Brown \& Ryan, 2003; Carmody \& Baer, 2008; Jain et al., 2007; Nyklíček \& Kuijpers, 2008; Shapiro, Oman, Thoresen, Plante, \& Flinders, 2008; Wolkin, 2015).

\section{Referências}

Alberts, H. J. E. M. \& Thewissen, R. (2011). The Effect of a Brief Mindfulness Intervention on Memory for Positively and Negatively Valenced Stimuli. Mindfulness, 2(2), 73-77. https://doi.org/10.1007/s12671-011-0044-7

Baer, R. A., Smith, G. T., Hopkins, J., Krietemeyer, J., \& Toney, L. (2006). Using self-report assessment methods to explore facets of mindfulness. Assessment, 13(1), 27-45. https://doi.org/10.1177/1073191105283504

Baer, R. A., Smith, G. T., Lykins, E., Button, D., Krietemeyer, J., Sauer, S., .., \& Williams, J. M. G. (2008). Construct validity of the Five Facet Mindfulness Questionnaire in meditating and nonmeditating samples. Assessment, 15(3), 329-342. https://doi.org/10.1177/1073191107313003

Barros, V. V. de, Kozasa, E. H., Souza, I. C. W. de, \& Ronzani, T. M. (2014). Validity evidence of the brazilian version of the five facet mindfulness questionnaire (FFMQ). Psicologia: Teoria e Pesquisa, 30(3), 317-327. https://doi. org/10.1590/S0102-37722014000300009 
Bishop, S. R., Lau, M., Shapiro, S., Carlson, L., Anderson, N. D., Carmody, J., ..., \& Devins, G. (2004). Mindfulness: A proposed operational definition. Clinical Psychology: Science and Practice, 11(3), 230-241. https://doi.org/10.1093/ clipsy/bph077

Brown, K. W. \& Ryan, R. M. (2003). The benefits of being present: mindfulness and Its role in psychological well-being. Journal of Personality and Social Psychology, 84(4), 822-848. https://doi.org/10.1037/0022-3514.84.4.822

Campbell, T. S., Labelle, L. E., Bacon, S. L., Faris, P., \& Carlson, L. E. (2012). Impact of Mindfulness-Based Stress Reduction (MBSR) on attention, rumination and resting blood pressure in women with cancer: a waitlist-controlled study. Journal of Behavioral Medicine, 35(3), 262-271. https://doi.org/10.1007/s10865-011-9357-1

Carmody, J. \& Baer, R. A. (2008). Relationships between mindfulness practice and levels of mindfulness, medical and psychological symptoms and well-being in a mindfulness-based stress reduction program. Journal of Behavioral Medicine, 31, 23-33. https://doi.org/10.1007/s10865-007-9130-7

Chambers, R., Yee Lo, B. C., \& Allen, N. B. (2008). The impact of intensive mindfulness training on attentional control, cognitive style, and affect. Cognitive Therapy and Research, 32, 303-322. https://doi.org/10.1007/s10608-0079119-0

Dahl, C. J., Lutz, A., \& Davidson, R. J. (2015). Reconstructing and deconstructing the self: cognitive mechanisms in meditation practice. Trends in Cognitive Sciences, 19(9), 515-523. https://doi.org/10.1016/j.tics.2015.07.001

de Bruin, E. I., Topper, M., Muskens, J. G. A. M., Bögels, S. M., \& Kamphuis, J. H. (2012). Psychometric properties of the Five Facets Mindfulness Questionnaire (FFMQ) in a meditating and a non-meditating sample. Assessment, 19(2), 187-197. https://doi.org/10.1177/1073191112446654

Evans, D. R., Baer, R. A., \& Segerstrom, S. C. (2009). The effects of mindfulness and self-consciousness on persistence. Personality and Individual Differences, 47(4), 379-382. https://doi.org/10.1016/j.paid.2009.03.026

Feldman, G., Dunn, E., Stemke, C., Bell, K., \& Greeson, J. (2014). Mindfulness and rumination as predictors of persistence with a distress tolerance task. Personality and Individual Differences, 56. 154-158. https://doi.org/10.1016/j. paid.2013.08.040

Hilt, L. M. \& Pollak, S. D. (2012). Getting Out of Rumination: Comparison of Three Brief Interventions in a Sample of Youth. Journal of abnormal child psychology, 40(7), 1157-1165. https://doi.org/10.1007/s10802-012-9638-3

Jain, S., Shapiro, S. L., Swanick, S., Roesch, S. C., Mills, P. J., Bell, I., \& Schwartz, G. E. R. (2007). A randomized controlled trial of mindfulness meditation versus relaxation training: effects on distress, positive states of mind, rumination and distraction. Annals of Behavioral Medicine, 33(1), 11-21. https://doi.org/10.1207/s15324796abm3301_2

Kiken, L. G. \& Shook, N. J. (2014). Does mindfulness attenuate thoughts emphasizing negativity, but not positivity? Journal of research in personality, 53, 22-30. https://doi.org/10.1016/j.jrp.2014.08.002

Lutz, A., Dunne, J. D., \& Davidson, R. J. (2007). Meditation and the neuroscience of consciousness: An introduction. In P. Zelazo, M. Moscovitch, \& E. Thompson (Orgs.). Cambridge Handbook of Consciousness (p. 499-554). New York: Cambridge University Press. https://doi.org/10.1017/CBO9780511816789.020

Menezes, C. B. \& Dell'Aglio, D. D. (2009). Os efeitos da meditação à luz da investigação científica em psicologia: Revisão de literatura. Psicologia: Ciência e Profissão. 29 (2). 276-289. https://doi.org/10.1590/S1414-98932009000200006

Menezes, C. B. \& Dell'Aglio, D. D. (2010). The relationship between the practice of sitting and silent meditation, and psychological well-being, and the effects of personality traits. Interamerican Journal of Psychology, 44(1), 140-149. (DOI inexistente).

Menezes, C. B., Dell’Aglio, D. D., \& Bizarro, L. (2012). Meditação, bem-estar e a ciência psicológica: revisão de estudos empíricos. Interação em Psicologia, 15(2). 239-248. https://doi.org/10.5380/psi.v15i2.20249

Nyklíček, I. \& Kuijpers, K. F. (2008). Effects of mindfulness-based stress reduction intervention on psychological wellbeing and quality of life: Is increased mindfulness indeed the mechanism? Annual Behavioral Medicine, 35, 331-340. https://doi.org/10.1007/s12160-008-9030-2

Paul, N. A., Stanton, S. J., Greeson, J. M., Smoski, M. J., \& Wang, L. (2013). Psychological and neural mechanisms of trait mindfulness in reducing depression vulnerability. Social Cognitive and Affective Neuroscience, 8(1), 56-64. https://doi.org/10.1093/scan/nss070

Ramel, W., Goldin, P. R., Carmona, P. E., \& McQuaid, J. R. (2004). The Effects of Mindfulness Meditation on Cognitive Processes and Affect in Patients with Past Depression. Cognitive Therapy and Research, 28(4), 433-455. https://doi. org/10.1023/B:COTR.0000045557.15923.96

Sauer, S., Walach, H., Schmidt, S., Hinterberger, T., Lynch, S., Büssing, A., \& Kohls, N. (2013). Assessment of Mindfulness: Review on State of the Art. Mindfulness, 4(1), 3-17. https://doi.org/10.1007/s12671-012-0122-5

Sedlmeier, P., Eberth, J., Schwarz, M., Zimmermann, D., Haarig, F., Jaeger, S., \& Kunze, S. (2012). The psychological effects of meditation: a meta-analysis. Psychological Bulletin, 138(6), 1139-1171. https://doi.org/10.1037/a0028168

Shapiro, S. L., Oman, D., Thoresen, C. E., Plante, T. G., \& Flinders, T. (2008). Cultivating mindfulness: Effects on wellbeing. Journal of Clinical Psychology, 64(7), 840-862. https://doi.org/10.1002/jclp.20491

Siegling, A. B. \& Petrides, K. V. (2014). Measures of trait mindfulness: Convergent validity, shared dimensionality, and linkages to the five-factor model. Quantitative Psychology and Measurement, 5, 1-8. https://doi.org/10.3389/ fpsyg.2014.01164

Soler, J., Cebolla, A., Feliu-Soler, A., Demarzo, M. M. P., Pascual, J. C., Baños, R., \& García-Campayo, J. (2014). Relationship between Meditative Practice and Self-Reported Mindfulness: The MINDSENS Composite Index. PloS one, 9(1), 1-7. https://doi.org/10.1371/journal.pone.0086622 
Treynor, W., Gonzalez, R., \& Nolen-Hoeksema, S. (2003). Rumination Reconsidered: A Psychometric Analysis. Cognitive Therapy and Research, 27(3), 247-259. https://oi.org/10.1023/A:1023910315561

Vandenberghe, L. \& Assunção, A. B. (2009). Concepções de mindfulness em Langer e Kabat-Zinn: um encontro da ciência Ocidental com a espiritualidade Oriental. Contextos Clínicos, 2(2), 124-135. https://doi.org/10.4013/ctc.2009.22.07

Walsh, R. \& Shapiro, S. L. (2006). The meeting of meditative disciplines and western psychology: A mutually enriching dialogue. American Psychologist, 61(3), 227-239. https://doi.org/10.1037/0003-066X.61.3.227

Wolkin, J. R. (2015). Cultivating multiple aspects of attention through mindfulness meditation accounts for psychological well-being through decreased rumination. Psychology Research and Behavior Management, 8, 171-180. https://doi. org/10.2147/PRBM.S31458

Zanon, C. \& Teixeira, M. (2006). Adaptação do Questionário de Ruminação e Reflexão (QRR) para estudantes universitários brasileiros. Interação em Psicologia, 10(1). 75-82. https://doi.org/10.5380/psi.v10i1.5771

\section{Declaração:}

Todos os autores declaram que não possuem conflito de interesses neste trabalho.

Autores:

Francisco Crauss - Mestre, Universidade do Vale do Rio dos Sinos.

Carolina Baptista Menezes - Doutora, Universidade do Rio Grande do Sul.

Lucas Nascimento - Graduando, Universidade Federal de Santa Catarina.

Ilana Andretta - Doutora, Universidade do Vale do Rio dos Sinos.

Endereço para correspondência

Carolina B. Menezes

Universidade Federal de Santa Catarina

Departamento de Psicologia - Centro de Filosofia e Ciências Humanas

Campus Universitário - Trindade

88040970 - Florianópolis, SC, Brasil

$<$ menezescarolina@hotmail.com>

Recebido em: 28.02.2017

Aceito em: 31.03.2017 